\title{
Analytic Approach for 2D Phased Array Delay Law Calculation in Case of Inspection of Objects with Complex Geometry
}

\author{
Tautvydas Fyleris $^{1,2}$, Elena Jasiuniene ${ }^{1,3}$ \\ ${ }^{1}$ Prof. K. Baršauskas Ultrasound Research Institute, Kaunas University of Technology, \\ K. Baršausko St. 59, LT-51423 Kaunas, Lithuania \\ ${ }^{2}$ Department of Software Engineering, Kaunas University of Technology, \\ Studentu St. 50, LT-51368 Kaunas, Lithuania \\ ${ }^{3}$ Department of Electronics Engineering, Kaunas University of Technology, \\ Studentu St. 50, LT-51368 Kaunas, Lithuania \\ tautvydas.fyleris@ktu.lt
}

\begin{abstract}
Objects with complex geometry need to be inspected quickly and reliably, therefore it is not practical to use fixed focus transducer, because of wave propagation effects. To mitigate the problem with the irregular shape, it is possible to use a phased array with applied delay laws. For calculating required delays, it is required to have an object model with known surface points and have an algorithm which calculates. This paper presents computing friendly analytic method which could be used in the case when the object model is given by lines or it is possible to calculate surface tangents. In this paper is assumed that geometry is approximated with B-splines. Results of the algorithm are verified with finite element method.
\end{abstract}

Index Terms-Ultrasound; Phased array; Delay law optimization; Focus; Complex geometry; Numerical simulation.

\section{INTRODUCTION}

Conventional ultrasound systems, typically phased array transducers have independent control channels for separate elements of the transducer, which performs transmission signal control and received signal amplification. This allows to perform delay-and-sum (DAS) or more recently delaymultiply-and-sum (DMAS) [1], [2] signal beam-forming. Obviously, system imaging performance depends on used hardware: transducer parameters (Fig. 1) [3], analogue front-end performance, and on model information of the inspected object. For planar surfaces (when it is possible to express surface in analytic form, for example, object is bounded with straight surfaces) focusing is almost solved problem [4], but if surface has non regular complex form, solving delay law problem is not straightforward.

The main idea of focusing comes from the Snell's law, which is based on the shortest path principle (Fermat principle). It states that if material in which the sound speed is $c_{1}$ has boundary with another material in which sound speed is $c_{2}$ and angle of beam incidence is known, the refracted angle can be calculated using trigonometric functions; knowing the wave path it is possible to illuminate region of interest which is achieved by steering (delaying) ultrasonic radiation where phased shows its advantages to single element systems.

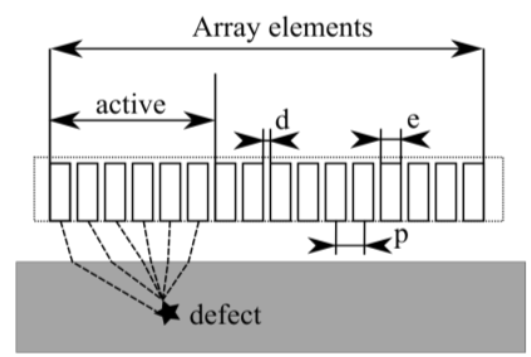

Fig. 1. Phased array example with the object and all components. It can be described by array size, $\mathrm{p}$ - pitch, e - element size, $\mathrm{d}$ - the distance between elements and have active elements number.

The objective of this work was - to create effective method for calculating optical path when there is only one boundary between two objects.

\section{Methodology}

Wave propagation mechanism has been the object of scientific research for years. Although at the first glance the problem seems to be relatively non-challenging and straightforward, due to non-regular geometry it becomes a complex problem possible to be solved only by finite element methods, which require long computational time. For faster results multiple less accurate methods where developed such as Pencil method employed in CIVA software [5] and multiple methods based on impulse response approach [6], [7] and of course for simple cases propagation can be calculated by classical wave propagation methods.

In this paper derivation process is analysed with the assumption that the object can be approximated by two types of surface elements: planar lines (Fig. 2(a)) as the most natural and computationally cheapest method; and bsplines which demands more computing resources (Fig. 2(b)), by fulfilling needed functionality on testing methods. 
Naturally, another surface approximation types exist, like NURBS surfaces, piecewise polynomials or analytical representations for every kind of surface. Current derivation is based on assumption that transducer and its travel path can be changed into infinite transducer with elements along scanning path (Fig. 2).

Beamforming of focusing problem is performed by exciting transducer elements with different time delays. Delay from source $\mathrm{S}$ (phased array element) to target $T$ (defect) was calculated using conventional methods and trigonometric functions with respect to angles or by Fermat principle to express as the shortest path problem [8]. Proposed method focusing is expressed by semi analitical fashion with coordinate transformation.

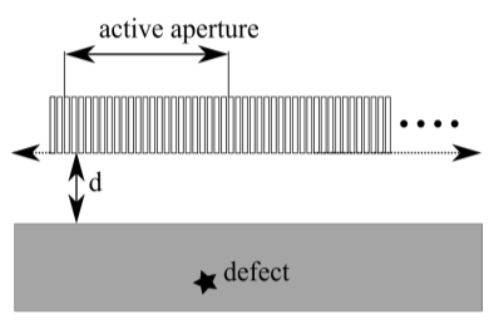

(a)

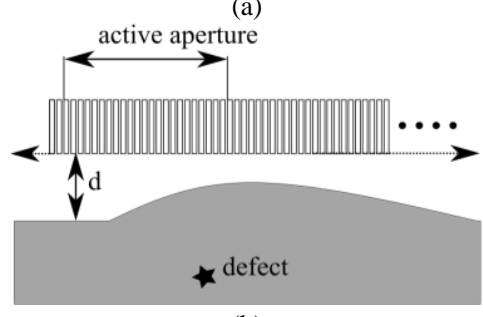

(b)

Fig. 2. Problem example with phased array being distance d with scanning line: $a$ - having planar surfaces it is not tricky to calculate focus point; $b$ from algorithmic point of view real transducer can be transformed into transducer having infinite elements with fixed aperture (in this paper it will be called scan line).

The path calculation problem can be expressed by five points (Fig. 3): $P$ - boundary line where the ray from the source elements intersects with the material (line length is always 1), $S$ - source point, $T$ - target point, $R-$ the intersection between normal from $\mathrm{P}$ point and direction line from $S$ point, $Z$ - intersection between normal from $P$ point and direction line from $T$ point.

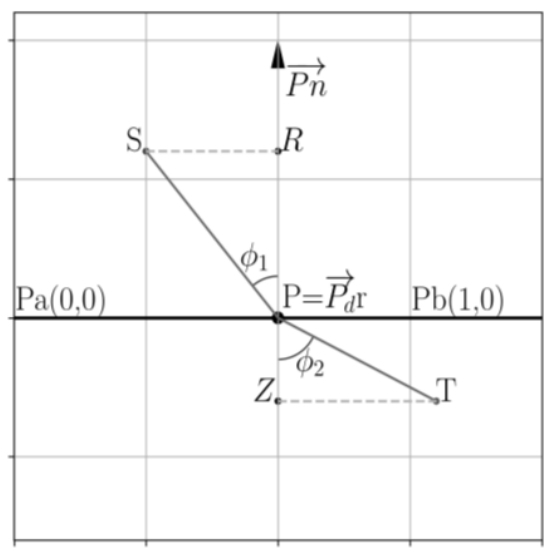

Fig. 3. Principal scheme of the equation in normalized units.

Direction vector (parametric normalized line equation) of the boundary point $P$ where the ray intersects with material has to be calculated:

$$
\begin{aligned}
& P d_{x}=P b_{x}-P a_{x}, \\
& P d_{y}=P b_{y}-P a_{y} .
\end{aligned}
$$

Two triangles SRP and TZP (Fig. 3) can be expressed according to the basic equations as a classic refraction problem. After inserting all variables, the following formulas were obtained:

$$
\begin{aligned}
& \sin \left(\varphi_{1}\right)= \\
& =\frac{\sqrt{P d_{x}^{2} s_{r}^{2}+P d_{y}^{2} s_{r}^{2}}}{\sqrt{\left(P d_{x} s_{r}+P o_{x}-S_{x}\right)^{2}}+\left(P d_{y} s_{r}+P o_{y}-S_{y}\right)^{2}}, \\
& \sin \left(\varphi_{2}\right)= \\
& =\frac{\sqrt{P d_{x}^{2} s_{z}^{2}+P d_{y}^{2} s_{z}^{2}}}{\sqrt{\left(P d_{x} s_{z}+P o_{x}-S_{x}\right)^{2}}+\left(P d_{y} s_{z}+P o_{y}-S_{y}\right)^{2}},
\end{aligned}
$$

where $s_{r}$ and $s_{z}$ are point length from $S$ point (vectors). After combining (3) and (4) formulas and reducing variables, Snell's law formula with SageMath [9] mathematics software is simplified to the following equation

$$
\begin{gathered}
\frac{-\left(P d_{x}^{2} r_{x}^{2}+P d_{y}^{2} r_{z}^{2}\right) c_{1}^{2}}{\left(P d_{x} r+P o_{x}-T_{x}\right)^{2}+\left(P d_{y} r+P o_{y}-T_{y}\right)^{2}}+ \\
+\frac{\left(P d_{x}^{2} r_{r}^{2}+P d_{y}^{2} r_{r}^{2}\right) c_{2}^{2}}{\left(P d_{x} r+P o_{x}-S_{x}\right)^{2}+\left(P d_{y} r+P o_{y}-S_{Y}\right)^{2}}=0,
\end{gathered}
$$

where $r_{x}, r_{z}, r_{r}$ are supporting triangle vectors; most of the equation can be reduced because using normalized values gives a lot of reductions (point $P$ can have values between 0 and 1) and it makes formula fully constrained.

After coordinate system transformation and boundary renormalization, (5) can be rewritten as

$$
\frac{-\left(T_{x}-r\right)^{2} c_{1}^{2}}{\left(T_{x}-r\right)^{2}+T_{y}^{2}}+\frac{\left(S_{x}-r\right)^{2} c_{2}^{2}}{\left(S_{x}-r\right)^{2}+S_{y}^{2}}=0 .
$$

Based on the derivation above, the angle calculation problem is transformed to root finding problem for polynomial, as there will be no trigonometric function of Snell's law. Equation can be further reduced taking advantage of fact that $S, P, T$ points always have some distance between them; we can drop denominator after simplifying fractions from (6)

$$
\begin{gathered}
\left(\left(T_{x}-r\right)^{2}+T_{y}\right)\left(S_{x}-r\right)^{2} c_{2}^{2}- \\
-\left(\left(S_{x}-r\right)^{2}+S_{y}^{2}\right)\left(T_{x}-r\right) c_{1}^{2}=0 .
\end{gathered}
$$

As it is possible to see, there can be more than one solution which holds condition but has no physical meaning in this paper. For this purpose it needs to be checked that there is a line $R Z$ between points $S$ and $T$. It can be achieved 
by creating following formula

$$
\begin{gathered}
\alpha_{1} \operatorname{sgn}\left(S_{x}-r\right)+\alpha_{2} \operatorname{sgn}\left(S_{y}\right)+ \\
+\alpha_{1} \operatorname{sgn}\left(T_{x}-r\right)+\alpha_{2} \operatorname{sgn}\left(T_{y}\right)=0,
\end{gathered}
$$

where $\alpha_{1}$ and $\alpha_{2}$ are arbitrary non-zero constants which are not equal; unequal alphas are optimization trick employed to check whether the Pn (Fig. 3) line is between $S$ and $T$.

Having derived (7) and (8) for the object described by bspline and lines, solving is transformed to the shortest optical path problem and is straightforward. For example, possible propagation path is shown in Fig. 4 and optimization problem values (it is not always possible to find exact roots) are plotted in Fig. 5.

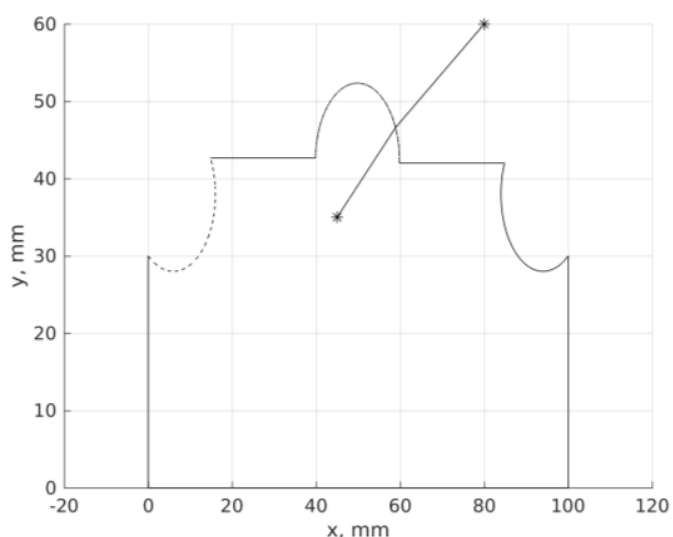

Fig. 4. Example shape with calculated point using the presented method.

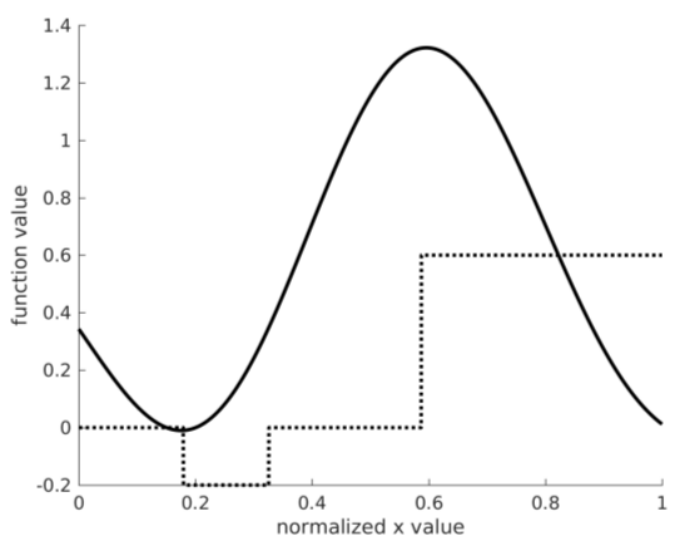

Fig. 5. Target function example: dashed line is check function with 0.1 and 0.3 alpha values, the solid line is an example of target function value of surface in Fig. 3 (please note that B-spline half circle approximation is used, function input varies from 0 to 1 , and there are two roots).

After finding the variable $r$ (point $P$ ) the transformation back to the original coordinate system is performed. As the intersection point is known, the beamforming delay calculation is nothing more but the calculation of acoustic path length. For example, $t_{i}$ is calculated according to the following equation

$$
\mathrm{t}_{\mathrm{i}}=\frac{\left|S_{i}-P_{i}\right|}{c_{1}}+\frac{\left|T_{i}-P_{i}\right|}{c_{2}} .
$$

\section{VERIFICATION}

For the verification model, FEM simulation is used. It is known that $100 \%$ accurate mapping cannot be created. The excitation signal of half sine wave is employed as a testing impulse (Fig. 6). There is no reason to use more complex signals because only wave extremum points are essential.

The most challenging part of this simulation is numerical dispersion [10] which can influence results. As only extremum points are essential, the dispersion due to an element size is not a significant problem. To verify this method, the following methodology was applied:

1. Multiple points of delay law on the scan line are calculated;

2. Simulation is performed;

3. Results from the target area are calculated using numerical calculations (because of dispersion and FEM element size only results from the area can be collected); 4. Stress values of wave $\mathrm{max} / \mathrm{min}$ times which depend on probe pulse shape are checked. In the current example; it is intervals of $0.5 \mu \mathrm{S}$.

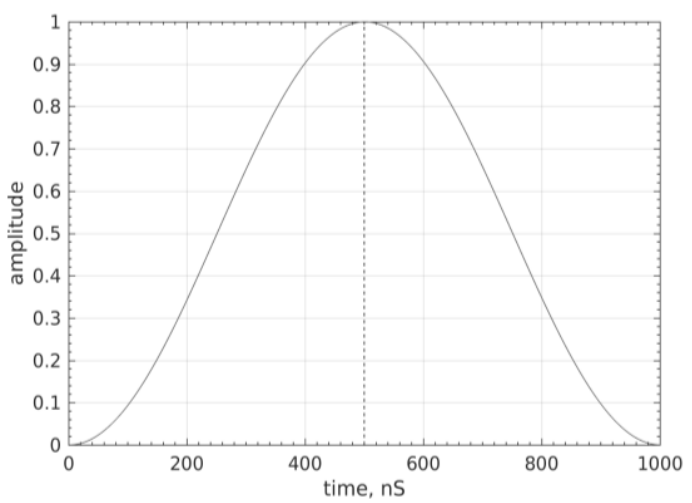

Fig. 6. Testing pulse with a normalized amplitude and half sine wave with its maximum is at $500 \mathrm{~ns}$.

It is a good idea to use not the first wave extremum point in checking but another - in the presented example the $4^{\text {th }}$ extreme point was used. The reason is that the first few wavefronts can be lost due to the dispersion.

\section{EXAMPLE}

The example demonstrating calculation of delay laws with two shape building blocks: line and B-Spline is provided in this chapter. For B-Spline used function target point is always 0 . Otherwise, optimization formula, which includes basis function, needs to be derived. It is not worth to derive it and for optimization of (7) and (8) iterative root finding method can be used from sage software [11]. The example below is calculated using iterative root finding algorithm method with the grid in the same way for line and B-splines. The result is demostrated in Fig. 6. The focus point is behind complex surface at $\mathrm{T}(45,35)$ from $\mathrm{S}(80,60)$. In order to calculate point on Figure 4 with conventional methods the shortest path algorithms could be employed [8], [12] results depend on the node size, using iterative algorithm and probing all pixels on boundary give same results, but computational price of lasting considerable time is high if pixels count is large.

The focusing to chosen point (area) delay law can be calculated by finding length between points and converting it into time and then offsetting results to longest element:

$$
t_{r}=\max t_{i},
$$




$$
t_{d i}=\left|t_{i}-t_{r}\right|
$$

where $t_{i}(10)$ is the longest distance between all active elements and target point, $t_{d i}(11)$ is delay law time. As we can see, the solution and delay law rule is quite simple.

Crosschecking for correct solution can be performed by the following steps: take multiple simulation points on trandcuer line (Fig. 2, choose time when wave has one of its extrema values (time of flight and transmitted signal already known) and check with theoretical signal interval (shown in Fig. 4). If there is a pattern with theoretical timings and interval, then it can be assumed that signal is traveling in a correct trajectory.

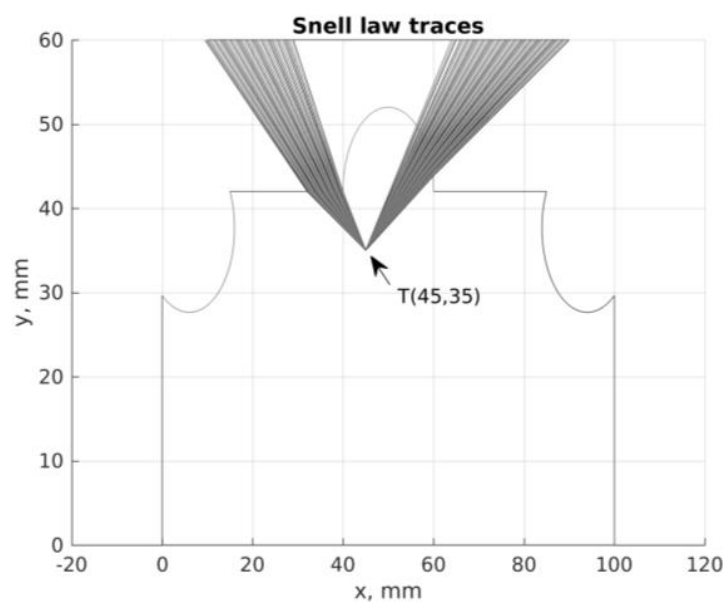

Fig. 7. Target and scanning line with hole in middle which means that their function value not converged.

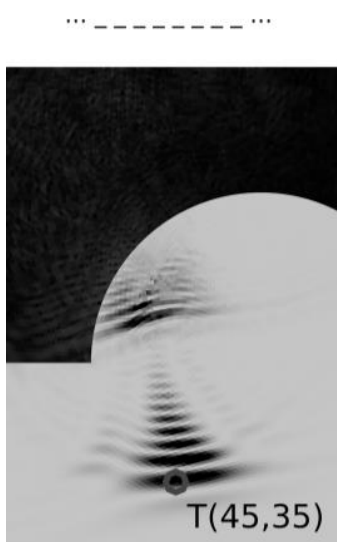

(a)

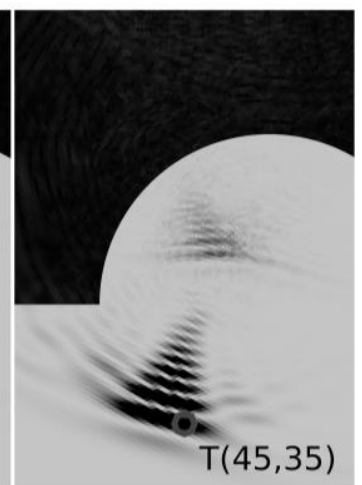

(b)
Fig. 8. FEM simulation results using Paraview [13] software to visualize, notice that target is a grey circle and shown time is same in both pictures, above picture is schematic transducer active focus taken from Fig. 7 from different focus laws $(a, b)$.

To check this method with FEM model simplified phased array model can be created (radiation elements are nodes at center of radiation element width). It is enough, in this case, to send half sin wave and check energy, which arrives at the target area. As it can be observed from the figure there is no need to have very accurate phased array model.

For verification simplified phased array model which includes only pressure lines on the system was used (posible pathes in Fig. 7). This lets us to create an uncomplicated testing workbench.

\section{CONCLUSIONS}

In this paper, a friendly approach for the calculation of delay law has been proposed. Only the multiplication operations are required in case of the planar surfaces. Choice of optimization function is not critical as results didn't change by selecting the functions provided by SciPy optimization package. Calculation with non-planar geometries depends on the b-spline approximation function because it is simpler and can approximate most of the physical objects. In future, authors propose to investigate NURBS surfaces by the presented method; authors plan to extend proposed method to work with more advanced focusing methodologies starting with classical total focusing method [14] and its variants.

\section{REFERENCES}

[1] G. Matrone, A. Ramalli, P. Tortoli, G. Magenes, "Experimental evaluation of ultrasound higher-order harmonic imaging with FilteredDelay Multiply And Sum (F-DMAS) non-linear beamforming", Ultrasonics, vol. 86, pp. 59-68, 2018. DOI 10.1016/j.sna.2018.01.002.

[2] G. Matrone, A. S. Savoia, G. Caliano, G. Magenes, "Ultrasound plane-wave imaging with delay multiply and sum beamforming and coherent compounding", in 2016 38th Annual Int. Conf. IEEE Engineering in Medicine and Biology Society (EMBC 2016), 2016, pp. 3223-3226. DOI: 10.1109/EMBC.2016.7591415.

[3] J. A. Jensen, N. B. Svendsen, "Calculation of pressure fields from arbitrarily shaped, apodized, and excited ultrasound transducers", IEEE Trans. Ultrason. Ferroelectr. Freq. Control, vol. 39, no. 2, pp. 262-267, 1992. DOI: 10.1109/58.139123.

[4] S. Chatillon, L. de Roumilly, J. Porre, C. Poidevin, P. Calmon, "Simulation and data reconstruction for NDT phased array techniques", Ultrasonics, vol. 44, pp. 951-955, 2006. DOI: 10.1016/j.ultras.2006.05.060

[5] S. R. Kolkoori, P. Shokouhi, C. Hoehne, M.-U. Rahman, M. Kreutzbruck, J. Prager, "A comparative study of ray tracing and CIVA simulation for ultrasonic examination of anisotropic inhomogeneous austenitic welds", AIP Conf. Proc., 2013, vol. 1511, no. 1, pp. 1043-1050. DOI: 10.1063/1.4789158.

[6] L. Aguilar, J. Wong, D. A. Steinman, R. S. C. Cobbold, "FAMUS II: A fast and mechanistic ultrasound simulator using an impulse response approach", IEEE Trans. Ultrason. Ferroelectr. Freq. Control, vol. 64, no. 2, pp. 362-373, 2017. DOI 10.1109/TUFFC.2016.2632706.

[7] S. Storve, H. Torp, "Fast Simulation of dynamic ultrasound images using the GPU”, IEEE Trans. Ultrason. Ferroelectr. Freq. Control, vol. 64, no. 10, pp. 1465-1477, 2017. DOI 10.1109/TUFFC.2017.2731944.

[8] O. Nowers, D. J. Duxbury, B. W. Drinkwater, "Ultrasonic array imaging through an anisotropic austenitic steel weld using an efficient ray-tracing algorithm", NDT E Int., vol. 79, pp. 98-108, 2016. DOI: 10.1016/j.ndteint.2015.12.009.

[9] S. Sage, the Sage Mathematics Software System (Version 7.4), 2016.

[10] A. Krisciunas, R. Barauskas, L. Mazeika, T. Fyleris, "Minimization of numerical dispersion errors in 2D finite element models of short acoustic wave propagation", Information and Software Technologies, pp. 745-752, 2016. DOI: 10.1007/978-3-319-46254-7_60.

[11] M. A. Gray, "Sage: A new mathematics software system", Comput. Sci. Eng., vol. 10, no. 6, pp. 72-75, 2008. DOI: 10.1109/MCSE.2008.154.

[12] H. Zhou, Z. Han, D. Du, Y. Chen, "A combined marching and minimizing ray-tracing algorithm developed for ultrasonic array imaging of austenitic welds", NDT E Int., vol. 95, pp. 45-56, 2018. DOI: 10.1016/j.ndteint.2018.01.008.

[13] A. Henderson, J. Ahrens, C. Law, The ParaView Guide. Kitware Clifton Park, NY, 2004.

[14] J. F. Cruza, J. Camacho, "Total focusing method with virtual sources in the presence of unknown geometry interfaces", IEEE Trans. Ultrason. Ferroelectr. Freq. Control, vol. 63, no. 10, pp. 1581-1592, 2016. DOI: 10.1109/TUFFC.2016.2593439. 